\title{
Temporal Correlators in the Continuous Time Formulation of Strong Coupling Lattice QCD
}

\author{
Marc Klegrewe* \\ Bielefeld University \\ E-mail: mklegrewe@physik.uni-bielefeld.de \\ Wolfgang Unger \\ Bielefeld University \\ E-mail: wungerephysik.uni-bielefeld.de
}

\begin{abstract}
We present results for lattice QCD in the limit of infinite gauge coupling on a discrete spatial but continuous Euclidean time lattice. A worm type Monte Carlo algorithm is applied in order to sample two-point functions which gives access to the measurement of mesonic temporal correlators. The continuous time limit, based on sending $N_{\tau} \rightarrow \infty$ and the bare anistotropy to infinity while fixing the temperature in a non-perturbative setup, has various advantages: the algorithm is sign problem free, fast, and accumulates high statistics for correlation functions. Even though the measurement of temporal correlators requires the introduction of a binning in time direction, this discretization can be chosen to be by orders finer compared to discrete computations. For different spatial volumes, temporal correlators are measured at zero spatial momentum for a variety of mesonic operators. They are fitted to extract the pole masses and corresponding particles as a function of the temperature. We conclude discussing the possibility to extract transport coefficients from these correlators.
\end{abstract}

The 36th Annual International Symposium on Lattice Field Theory - LATTICE2018

22-28 July, 2018

Michigan State University, East Lansing, Michigan, USA.

${ }^{*}$ Speaker. 


\section{Introduction}

The determination of the full QCD phase diagram, in particular the location of the critical point, is an important, long standing problem, requiring non-perturbative methods. In lattice QCD, several approaches have been developed to investigate the phase transition from the hadronic matter to the quark gluon plasma, but all of them are limited to small $\frac{\mu_{B}}{T}$ [1]. The reason for this is the notorious sign problem, which arises because the fermion determinant for finite baryon chemical potential $\mu_{B}$ becomes complex, and importance sampling is no longer applicable. In QCD, the sign problem is severe. Dual representations oftentimes solve or milden sign problems as being the case in strong coupling QCD (SC-QCD). Here, first the gauge degrees of freedom are integrated out exactly, which allows replacing the Grassman integration by a sum over fermionic color singlets, resulting in a partition function being expressed as a gas of hadron world lines (c.f. monomer-dimer system [2]). This representation allows us to obtain the full $\left(\mu_{B}, T\right)$ phase diagram, and it shares important features of QCD such as confinement and spontaneous chiral symmetry breaking and its restoration at a transition temperature $T_{c}$ [3]. Moreover, the chiral limit can be studied very economically - simulations are faster than with a finite quark mass. The Continuous Euclidean Time limit with its many assets (c.f. chap. 4) was first proposed to be applied to quantum field theories by Beard and Wiese [4]. Here, we use it to remove the sign problem completely.

\section{Strong Coupling QCD}

In SC-QCD, the gauge coupling is sent to infinity and hence the coefficient of the plaquette term $\beta=6 / g^{2}$ is sent to zero. Thus, the Yang Mills part $F_{\mu \nu} F_{\mu \nu}$ is absent. Subsequently, the gauge fields in the covariant derivative can be integrated out analytically. However, as a consequence of the SC-limit, the lattice spacing $a$ becomes very coarse, and no continuum limit can be achieved. We consider the SC-limit for staggered fermions. The final partition function for the discrete system on a $N_{\sigma}{ }^{3} \times N_{\tau}$ lattice, after performing the Grassmann integrals analytically, is given by

$$
\begin{gathered}
\mathscr{Z}\left(\gamma, N_{\tau}, m_{q}\right)=\sum_{\{k, n, \ell\}} \prod_{b=(x, \hat{\mu})} \frac{\left(N_{c}-k_{b}\right) !}{N_{c} ! k_{b} !} \gamma^{2 k_{b} \delta_{\hat{0}, \mu}} \prod_{x} \frac{N_{c} !}{n_{x} !}\left(2 a m_{q}\right)^{n_{x}} \prod_{l} w(\ell, \mu) \\
\text { Grassmann constraint: } \quad n_{x}+\sum_{\hat{\mu}= \pm \hat{0}, \ldots \pm \hat{d}}\left(k_{\hat{\mu}}(x)+\frac{N_{c}}{2}\left|\ell_{\mu}(x)\right|\right)=N_{c}, \quad \forall x \in N_{\sigma}^{3} \times N_{\tau} \\
w(\ell, \mu)=\sigma(\ell) \gamma^{N_{c} \sum_{x}\left|\ell_{0}(x)\right|} \exp \left(N_{c} N_{\tau} r(\ell) a_{\tau} \mu\right), \quad \sigma(\ell)=(-1)^{r(\ell)+N_{-}(\ell)+1} \prod_{b=(x, \hat{\mu}) \in \ell} \eta_{\hat{\mu}}(x)
\end{gathered}
$$

where $\gamma$ is the bare anisotropy coupling. After this exact rewriting of the strong coupling partition function the system is described by confined, colorless, discrete degrees of freedom:

- Mesonic degrees of freedom $k_{\hat{\mu}}(x) \in\left\{0, \ldots N_{c}\right\}$ (non-oriented meson hoppings called dimers) and $n(x) \in\{0, \ldots N c\}$ (mesonic sites called monomers).

- Baryonic degrees of freedom, which form oriented baryon loops $\ell$ with sign $\sigma(\ell)= \pm 1$ and winding number $r(\ell)$ that depend on the geometry of the loops Eq. (2.3). These loops are self-avoiding and do not touch the mesonic degrees of freedom.

Both mesonic and baryonic degrees of freedom obey the Grassmann constraint Eq. (2.2). Monomers are absent since we will restrict to the chiral limit $m_{q}=0$. 


\section{Anisotropic lattices}

On a bipartite lattice with staggered fermions an even number of lattice points is required in all directions. Thus, the highest temperature that is possible to be addressed on an isotropic lattice is $a T=$ $1 / N_{\tau}=0.5$, with $N_{\tau}$ the temporal extent. This is significantly too low to reach the critical temperature of chiral restoration. So, in practice, anisotropic lattices are chosen to study thermodynamical properties of staggered lattice QCD, in particular across the chiral phase transition. The anisotropy parameter $\xi=\frac{a}{a_{\tau}}$ is introduced into the definition of the lattice temperature

$$
T=\frac{1}{a_{\tau} N_{\tau}}=\frac{\xi(\gamma)}{a N_{\tau}}
$$

which allows to assign different extents in spatial and temporal direction and thus, to modify the temperature continuously even above the chiral transition. $\xi$ becomes unity when the lattice is isotropic and diverges in the CT limit $a_{\tau} \rightarrow 0$. As highlighted in Eq. (3.1) the anisotropy parameter depends on the bare anisotropy coupling $\gamma$. However, the exact functional correspondence is unknown. Recent non-perturbative studies [5] suggest that

$$
\xi(\gamma) \simeq \kappa \gamma^{2}+\frac{\gamma^{2}}{1+\lambda \gamma^{4}}, \quad \kappa=0.781(1) \text { for } \mathrm{SU}(3) .
$$

Further simplification is achieved by eliminating $\gamma$ and $N_{\tau}$, and to replace them by the temperature $a T$ completely. This is summarized in the continuum limit in Euclidean time:

$$
N_{\tau} \rightarrow \infty, \quad \gamma \rightarrow \infty, \quad \kappa \gamma^{2} / N_{\tau} \equiv a T \text { fixed }
$$

Here, $\kappa \gamma^{2} / N_{\tau}$ represents the temperature $a T$ in a well defined setup. Only one parameter is left that sets the thermal properties, and all discretization errors introduced by a finite $N_{\tau}$ are removed.

\section{Continuous Time Limit and worm algorithm}

Designing an algorithm that operates in the continuous time (CT) limit will have several advantages: Since there is no need to perform the continuum extrapolation $N_{\tau} \rightarrow \infty$, critical temperatures can be estimated more precisely, with a faster algorithm which only depends on one parameter, the temperature $\mathrm{T}$. Moreover, ambiguities arising from the functional dependence of observables on the anisotropy parameter will be circumvented. Also in the baryonic part of the partition function great simplifications occur: Baryons become static in the CT limit for $N_{c} \geq 3$, hence, the sign problem is completely absent. The CT partition function is obtained by the joined limit $\gamma$ and $N_{\tau} \rightarrow \infty$ and includes:

- dimer contributions of Eq. (2.1) are factorized into a spatial and temporal part and $\mathscr{Z}\left(\gamma, N_{\tau}\right)$ is rewritten such that spatial dimers obtain a weight $\gamma^{-2}[6]$.

- the limit $\gamma \rightarrow \infty$ implies that configurations with only zero or single spatial dimers contribute while configurations with multiple spatial dimers are considered to be suppressed.

- configurations are characterized fully by dimers in the zero time slice $k_{0}(0) \in\left\{0, \ldots N_{c}\right\}$ and bonds occupied by single spatial dimers which form vertices. Intervals between vertices have a weight of one and are omitted. 
- the limit $N_{\tau} \rightarrow \infty$ removes lattice artifacts in $a_{\tau}$ completely. Due to the even-odd decomposition there are $\frac{N_{\tau}}{2}$ positions available to distribute an oriented spatial dimer which gives rise to the factor $\frac{1}{2 a T}$ in Eq. (4.1).

Finally, a merely $\mathrm{T}$ dependent partition function is obtained

$$
\begin{aligned}
\left(N_{c}=3\right): \quad \quad \mathscr{Z}(T) & =\sum_{k \in 2 \mathbb{N}}\left(\frac{1}{2 a T}\right)^{k} \sum_{\mathscr{G}^{\prime} \in \Gamma_{k}} e^{\mu_{B} B / T} \hat{v}_{\perp}^{N_{\perp}} \quad \text { with } \hat{v}_{\perp}^{N_{\perp}}=2 / \sqrt{3} \\
\text { and } k & =\sum_{b=(x, \hat{i})} k_{b}=\frac{N_{\llcorner}+N_{\perp}}{2}, \quad N_{\llcorner/ \perp}=\sum_{x} n_{\llcorner/ \perp}(x)
\end{aligned}
$$

with the baryon number $B$, a non-trivial vertex weight $\hat{v}_{\perp}^{N_{\perp}}$, the number of $L / T$-shaped vertices $N_{\llcorner} / N_{\perp}$ and $\Gamma_{k}$ being the set of equivalence classes $\mathscr{G}^{\prime}$ of graphs containing a total number $k$ of spatial hoppings, equivalent up to time shifts of the vertices.

Now, to sample this CT partition function a worm type algorithm is used, similar to the directed path algorithm introduced for SC-QCD in [7]. In analogy to the decomposition of the lattice into active and passive sites, we decompose the lattice into emission and absorption sites. By definition the worm tail is located at an absorption site and violates Eq. (2.2). As a consequence, the worm head propagates through the lattice and restores (violates) the constraint in turns while visiting emission (absorption) sites respectively. During propagation the worm head either stops at an absorption site connected to a spatial dimer or emits a spatial dimer after some distance $\Delta \beta$ established by a Poisson process. The Poisson process assures that the oriented vertices, which always connect an emission and an absorption site, are exponentially distributed

$$
P(\Delta \beta)=\exp (-\lambda \Delta \beta), \quad \Delta \beta \in[0, \beta=1 / a T], \quad \lambda=d_{M}(x, t) / 4, \quad d_{M}(x, t)=2 d-\sum_{\hat{\mu}} n_{B}(x+\hat{\mu})
$$

with $\lambda$ the "decay constant" for spatial dimer emissions. Due to the presence of baryons, $\lambda$ is space-time dependent, with $d_{M}(x, t)$ being the number of mesonic neighbors at a given coordinate. Throughout the worm evolution monomer-monomer two-point correlation functions are accumulated whenever the Grassmann constraint is restored by taking into account the respective positions of worm tail and head:

$$
C\left(t_{H}-t_{T}, \vec{x}_{H}-\vec{x}_{T}\right)=C(\tau, \vec{x})=N_{c} \frac{O(C(\tau, \vec{x}))}{\text { \#worm updates }} .
$$

Such worm estimators are incremented as $O(C(\tau, \vec{x})) \rightarrow O(C(\tau, \vec{x}))+f(\ldots) \cdot \delta_{x_{T}, x_{1}} \delta_{x_{H}, x_{2}}$ with

$$
\begin{aligned}
\text { discrete time: } f(\gamma), & \tau \in\left[0, \ldots N_{\tau}\right] \\
\text { continuous time: } f(T), & \tau \in[0, \ldots 1 / T] .
\end{aligned}
$$

By summing over them yields immediately the chiral susceptibility:

$$
\chi_{\sigma, D T}=\frac{1}{V} \sum_{\vec{x}, \tau} C(\tau, \vec{x}) \quad \text { and } \quad \chi_{\sigma, C T}=\frac{1}{V} \sum_{\vec{x}} \int_{0}^{1 / T} d \tau C(\tau, \vec{x}) .
$$

\section{Temporal Correlators}

As for temporal correlators in CT, the increment $f(T)$ of Eq. (4.3) is spread out to bins across the path covered by the worm head in temporal direction. Thus, even for the $\mathrm{CT}$ algorithm a discretization is 
Table 1: The sign $g_{x}^{D}$ defined in Eq. (5.1) yields different correlators for the kernel $\left(\Gamma^{D} \otimes \Gamma^{F}, \Gamma^{D}=\Gamma^{F *}\right)$. Corresponding continuum and particle states for $N_{f}=1$ are named.

\begin{tabular}{c|cc|cc|cc}
\hline$g_{x}^{D}$ & \multicolumn{2}{|c|}{$\Gamma^{D} \otimes \Gamma^{F}$} & \multicolumn{2}{|c|}{$J^{P C}$} & \multicolumn{2}{|c}{ Physical states } \\
& $\mathrm{NO}$ & $\mathrm{O}$ & $\mathrm{NO}$ & $\mathrm{O}$ & $\mathrm{NO}$ & $\mathrm{O}$ \\
\hline 1 & $1 \otimes 1$ & $\gamma_{0} \gamma_{5} \otimes\left(\gamma_{0} \gamma_{5}\right)^{*}$ & $0^{++}$ & $0^{-+}$ & $\sigma_{S}$ & $\pi_{A}$ \\
$(-1)^{x_{i}}$ & $\gamma_{i} \gamma_{5} \otimes\left(\gamma_{i} \gamma_{5}\right)^{*}$ & $\gamma_{i} \gamma_{0} \otimes\left(\gamma_{i} \gamma_{0}\right)^{*}$ & $1^{++}$ & $1^{--}$ & $a_{A}$ & $\rho_{T}$ \\
$(-1)^{x_{j}+x_{k}}$ & $\gamma_{j} \gamma_{k} \otimes\left(\gamma_{j} \gamma_{k}\right)^{*}$ & $\gamma_{i} \otimes \gamma_{i}^{*}$ & $1^{+-}$ & $1^{--}$ & $b_{T}$ & $\rho_{V}$ \\
$(-1)^{x_{i}+x_{j}+x_{k}}$ & $\gamma_{0} \otimes \gamma_{0}^{*}$ & $\gamma_{5} \otimes\left(\gamma_{5}\right)^{*}$ & $0^{+-}$ & $0^{-+}$ & ${ }_{V}$ & $\pi_{P S}$ \\
\hline
\end{tabular}

introduced, however, it can be chosen by orders finer in comparison to a discrete time lattice extent. It is distinguished between two different histograms, either with even (absorption-absorption) or odd (absorptionemission) temporal distance contributions. Combinations of these histograms allow to construct correlators for the Non-Oscillating (NO) and Oscillating (O) channel. Additionally, by including the sign $g_{x}^{D}$ listed in Table 1 various states are addressed for $N_{f}=1$. Since temporal correlators at zero spatial momentum are measured, the extracted meson masses are pole masses $\left(E_{0}(\vec{p}=0)=m_{0}\right)$. The respective correlators are expressed as a sum over the staggered fermion fields $\bar{\chi}_{x} \chi_{x}$ with a diagonal dirac-taste kernel $\left(\Gamma^{D}=\Gamma^{F *}\right)$, that is realized by the signs $g_{x}^{D}$ :

$$
C(t)=\sum_{\vec{x}}\left\langle\bar{\chi}_{0} \chi_{0} \bar{\chi}_{\vec{x}, t} \chi_{\vec{x}, t}\right\rangle \cdot g_{x}^{D} .
$$

Hereafter, the workflow for continuous time pole mass extraction as well as discrete time is highlighted and results are compared. In order to obtain discrete time temporal correlators the even and odd histograms are fitted via a four parameter ansatz respectively:

$$
\begin{aligned}
& C_{\mathrm{DT}, \text { Even }}(\tau)=a_{N O} \cosh \left(m_{N O}\left(\tau-N_{\tau} / 2\right)\right)-a_{O} \cosh \left(m_{O}\left(\tau-N_{\tau} / 2\right)\right. \\
& C_{\mathrm{DT}, \text { Odd }}(\tau)=\underbrace{a_{N O} \cosh \left(m_{N O}\left(\tau-N_{\tau} / 2\right)\right)}_{\text {Non-oscillating Correlator }}+\underbrace{a_{O} \cosh \left(m_{O}\left(\tau-N_{\tau} / 2\right)\right.}_{\text {Oscillating Correlator }} .
\end{aligned}
$$

A combined fit to simultaneously describe both data sets is possible, but more challenging when it comes to fit convergence. Finally, by addition/subtraction the correlators are as follows:

$$
C_{\mathrm{DT}, \mathrm{NO}}(\tau)=\frac{1}{2}\left(C_{\mathrm{DT}, \mathrm{Even}}(\tau)+C_{\mathrm{DT}, \mathrm{Odd}}(\tau)\right), \quad C_{\mathrm{DT}, \mathrm{O}}(\tau)=\frac{1}{2}\left(C_{\mathrm{DT}, \mathrm{Even}}(\tau)-C_{\mathrm{DT}, \mathrm{Odd}}(\tau)\right) .
$$

Note that in discrete time only histogram data sets are described by the fits as presented in Fig. (1a), however, the final constructed correlators are not. Now, these fits have to be performed for various $N_{\tau}$ (c.f. Fig. (1b)) such that an appropriate $N_{\tau} \rightarrow \infty$ extrapolation can be carried out (c.f. Fig. (1d)). Finally, this workflow is necessary for the different channels and multiple temperatures. In comparison, the added and subtracted histograms out of continuous time simulations

$$
\begin{gathered}
C_{\mathrm{CT}, \mathrm{O}}(\tau)=a_{N O} \cosh \left(m_{N O}(\tau-1 / 2)\right)=\frac{1}{2}\left(C_{\mathrm{Odd}}(\tau)+C_{\mathrm{Even}}(\tau)\right) \\
C_{\mathrm{CT}, \mathrm{NO}}(\tau)=a_{O} \cosh \left(m_{O}(\tau-1 / 2)\right)=\frac{1}{2}\left(C_{\mathrm{Odd}}(\tau)-C_{\mathrm{Even}}(\tau)\right)
\end{gathered}
$$

give directly rise to the (Non)-Oscillating correlators respectively and are fitted in accordance with Eq. (5.4) as shown in Fig. (1c). The resulting pole masses are measured in $M / T$. Finally, Fig. (2a) shows a com- 


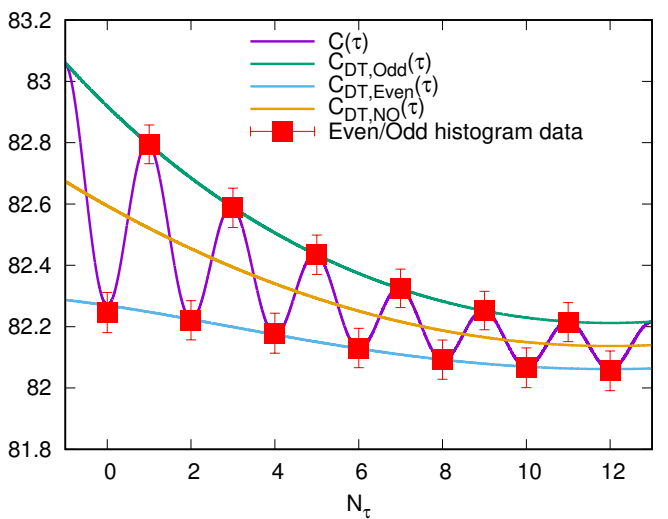

(1a) Histogram data fitted by several approaches, leading to the constructed correlator $C_{D T, N O}(\tau)$.

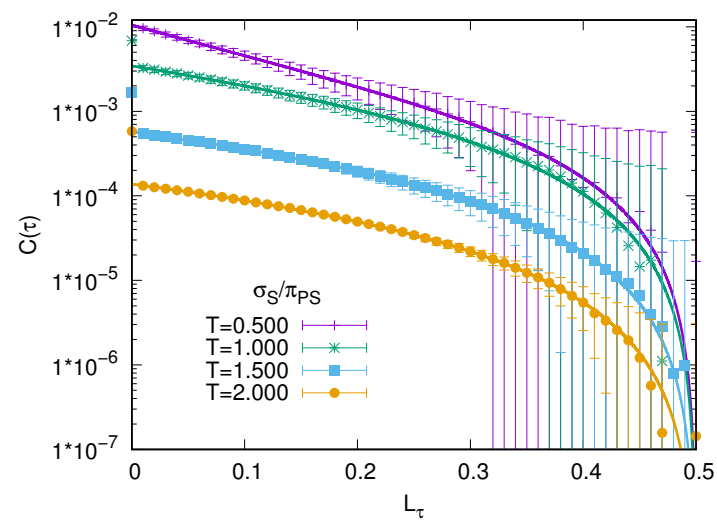

(1c) Continuous time histogram data fitted according to Eq. (5.4) for the mass degenerated channels $\sigma_{S} / \pi_{P S}$.

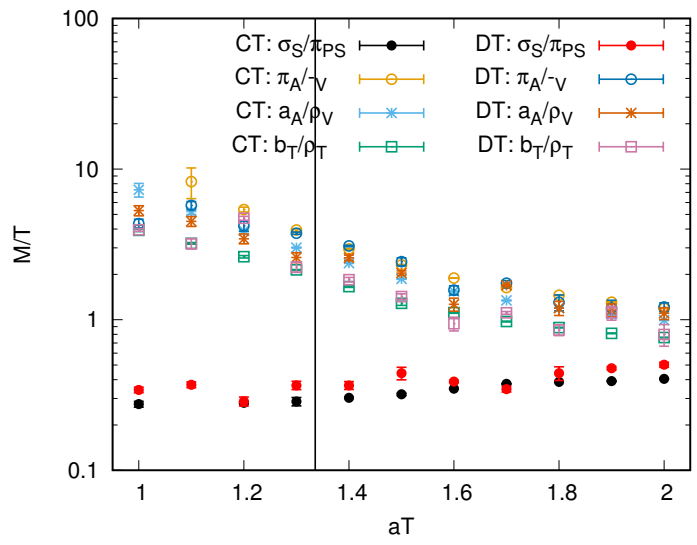

(2a) $M / T$ for different temepratures $a T$ and channels measures either in CT or DT. Certain channels listed in (5.5) are mass degenerated. Agreement between $\mathrm{CT}$ and DT is visible.

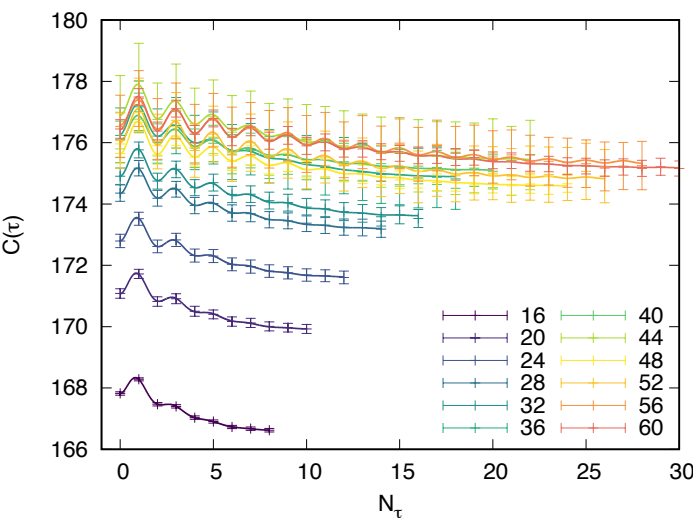

(1b) Histogram data fits for multiple temporal extension ranging from $N_{\tau}=16$ to $N_{\tau}=60$.
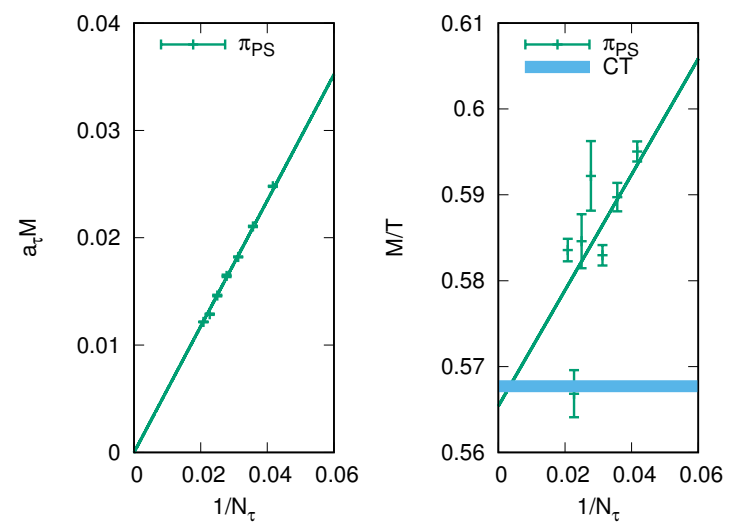

(1d) Extrapolated masses from discrete time simulations. Rescale to units of $M / T$ and compare with continuous time simulation result (blue band).

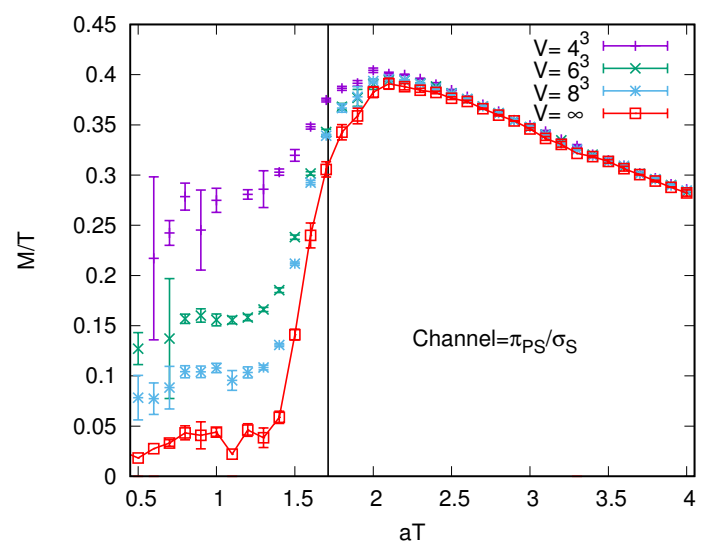

(2b) $M / T$ over $a T$ for different volumes but the same channel $\pi_{P S} / \sigma_{S}$. The continuum extrapolated red curve supports the expectations to have a zero mass below $T_{c}$ for simulations in the chiral limit. 
parison of the extrapolated masses. For SU(3) the chiral transition is located at $a T \approx 1.403$ where indeed an impact on the masses is obtained. So far, only a small temperature range $a T=\{1.0, \ldots 2.0\}$ is studied since especially small temperatures are expensive and simulations with reliable outcome are presented in the future. Simulations performed in discrete and continuous time give consistent results. Currently, there are still larger fluctuations and errorbars present in the discrete data. On the contrary, continuous time results have a smoother behavior down to the chiral transition but then more statistics and simulation time is needed. For $N_{f}=1$ we find a mass degeneracy for the channel pairs:

$$
\sigma_{S} \leftrightarrow \pi_{P S} \quad \pi_{A} \leftrightarrow-_{V} \quad b_{T}\left(\gamma_{j} \gamma_{k}\right) \leftrightarrow \rho_{T}\left(\gamma_{i} \gamma_{0}\right) \quad a_{A}\left(\gamma_{i} \gamma_{5}\right) \leftrightarrow \rho_{V}\left(\gamma_{i}\right) .
$$

Since simulations are performed in the chiral limit in a finite volume ( $\varepsilon$--regime) the mass degeneracy of the Non-Oscillating scalar channel with the Oscillating pseudo-scalar channel is expected. Due to finite volume effects non-zero $M / T$ values are obtained also in the regime below $T_{c}$, however, a first continuum extrapolation in Fig. (2b) clearly corrects this towards $M / T \rightarrow 0$.

\section{Conclusion}

With the CT worm algorithm we measured monomer-monomer two-point correlation functions and constructed temporal correlators with projected zero spatial momentum. For a temperature range around the chiral transition we obtain consistent pole mass results for discrete and continuous time simulations. Due to simulations being tremendously more expensive for small temperature further analysis is in progress.

The zero momentum meson correlators can be used to calculate the diffusion constant by extracting the spectral function from the correlation data applying standard methods like MEM. In continuous time we profit from being able to choose the temporal discretization by orders finer compared to discrete time computations.

Future calculations will be performed for finite quark masses, multiple flavors by making use of a Hamiltonian formulation [8] which will control and remove the sign problem and finally by including $\beta$ corrections to move away from the strong coupling limit.

\section{Acknowledgments}

Numerical simulations were performed on the OCuLUS cluster at PC2 (Universität Paderborn). We acknowledge support by the Deutsche Forschungsgemeinschaft (DFG) through the Emmy Noether Program under Grant No. UN 370/1 and through the Grant No. CRC-TR 211 "Strong-interaction matter under extreme conditions".

\section{References}

[1] P. de Forcrand, PoS (LAT2009) 010 (2009), arXiv:1005.0539.

[2] P. Rossi, U. Wolff, Nucl. Phys. B 248 (1984) 105.

[3] P. de Forcrand, M. Fromm, Phys. Rev. Lett. 104 (2010) 112005.

[4] B. B. Beard and U.-J. Wiese, Phys. Rev. Lett. 77 (1996) 5130.

[5] P. de Forcrand, W. Unger, H. Vairinhos (2017), arXiv:1710.00611.

[6] W. Unger, P. de Forcrand, PoS (LAT2011) 218 (2011), 1111.1434

[7] D. H. Adams, S. Chandrasekharan, Nucl. Phys. B 662 (2003) 220.

[8] W. Unger and P. de Forcrand, PoS (LAT2012) 194 (2012), arXiv:1211.7322. 